\title{
The effect of dietary fibers on the viscoelastic properties of the gluten-free cookie dough
}

\author{
Irina Krasina*, Anna Kurakina, Elena Viktorova, and Anastasia Strelkova \\ Kuban State Technological University, 2 str. Moskovskaya, Krasnodar, 350072, Russia
}

\begin{abstract}
The significance of inclusion of associated dietary fibres in various amounts to the formulations of gluten-free rice test mixtures based on rheological profile of hydrated fibrous-flour composite mixtures was investigated. To assess the viscoelasticity of fiber-enriched rice-based test matrices, dual fundamental (dynamic oscillatory and creep recovery tests) and empirical (consistency and viscometric profile) rheological approaches were adopted. The obtained functional variables were analyzed for the dependence on the hydration of the dough and dietary fibres, as well as for the correlations within the parameters of both small and large deformation tests. The aqueous competition of fiber macromolecules, exhibiting different water binding and gelling capacities, led to additive, synergistic and/or antagonistic effects on the basic rheological properties.
\end{abstract}

Celiac disease is an immune-mediated enteropathy caused by the gluten ingestion in genetically susceptible individuals. Celiac disease is one of the most common human genetic diseases with a prevalence of $1-2 \%$ worldwide, the frequency of which is growing [1]. Despite the advances in celiac disease pathogenesis understanding and the potential development of new therapies, currently the only safe and effective treatment for the patients with this disease is lifelong avoidance of gluten-containing foods. One of the main challenges that people with celiac disease can fully adhere to a gluten-free diet is finding good quality gluten-free foods. Despite the fact that the several gluten-free products are currently available on the market, gluten-free baking ingredients, as a rule, have low physicochemical and sensory quality and have low content of fiber, vitamins and some other nutrients, which leads to the condition deterioration on the already weakened with unbalanced diet body of patients. Although the development of functional food products for a healthy population is now a high priority, little attention is paid to products for people with celiac disease. The fortification of flour confectionery with dietary fibres with established physiological advantages is of particular interest to this population, which has a significant incidence of certain comorbidities such as diabetes [2-4].

In the confectionery industry, the careful selection of dietary fibers with suitable physicochemical properties that contribute to irreversible degradation of the protein matrix, which includes excessive weakening of protein / starch networks, is a prerequisite for obtaining the workable dough, especially for gluten-free systems without an endogenous viscoelastic biopolymer.

\footnotetext{
* Corresponding author: kib6060@mail.ru
} 
The consumption of fructans such as inulin and oligofructose has been investigated as a resource for increasing dietary fiber content in gluten-free cookies $[5,6,7]$. Inulin is a polysaccharide with interesting functional properties and probiotic activity. It is synthesized as a storage carbohydrate in many cultivated plants. Due to its chemical structure, inulin cannot be absorbed in the digestive tract, and products with its share are characterized by reduced energy value. In addition to these health benefits, inulin may play a functional role in the food industry. It is used as a filler and structure-forming agent, as well as a substance that improves rheological properties and taste [8-11]. Various hydrocolloids can be used to imitate the viscoelastic properties of gluten [12-14 ]. Significant viscosity changes that occur when gums are added are of great industrial importance.

A mixture of dietary fibers consisting of inulin, oligofructose and xanthan gum was obtained for the preparation of cookies, the ratio of components was determined by preliminary studies [15]. This mixture of dietary fibers was substituted for 25,50 and $75 \%$ of the rice flour.

Dual fundamental and empirical rheological approaches have been used to assess the viscoelasticity of food fiber-enriched rice-based dough matrices. Dynamic fluctuations and rheological creep recovery characteristics, consistency and viscosimetric profile were evaluated in a gluten-free dough according to the Draper-Lin plan. The obtained instrumental physical parameters were analyzed for dependence on the dough hydration and the dietary fibers viscosity, as well as for the correlations presence within the parameters from tests for small and large deformations.

In determining of the dietary fibers effect on the rheological properties of the gluten-free dough, the studies for establishing the levels of various design factors were conducted. Analytical data on the fundamental and empirical rheological characteristics of the test were adapted to multiple regression equations using additional principles as independent factors to estimate the response of the dependent analytical variables.

In fundamental measurements, the viscoelasticity of the gluten-free test was checked by vibration and creep measurements. Oscillatory measurements of the linear viscoelastic region, performed without disturbing or destroying the internal structure, have proved useful in studying the effects and values of structural ingredients, such as hydrocolloids, in disperse dough systems, since dynamic mechanical parameters are very sensitive to changes in the type and concentration of the polymer, as well as to the water content.

The linear viscoelastic region for gluten-free dough samples was established by voltage scanning experiments from 0 to $60 \mathrm{~Pa}$. The drop in the modulus of elasticity, $\mathrm{G}$ ', began to occur at voltage values that ranged from $3 \mathrm{~Pa}$ to more than $60 \mathrm{~Pa}$, indicating different tensile strengths resistance for gluten-free dough structures. The samples with the highest water content had the lowest limit voltage in the linear viscoelastic region. The Dough with low water content showed a broader linear viscoelastic region. The correction of values of dynamic modulus of elasticity and viscosity depending on frequency $(0.1-10 \mathrm{~Hz})$ into the equation of potential made it possible to estimate $\mathrm{G}_{1}$ ' and $\mathrm{G}_{1}$ " at the same time the values of R2 ranged from 0.99 to 0.9999 .

The G1 'and G1 " coefficients were significantly dependent on the presence of the dietary fiber blend. The single addition of hydrocolloids to varying degrees contributed to an increase in modulus of elasticity and viscosity. The addition of inulin had a great impact, especially on the G1 ' elasticity modulus through quadratic and linear positive effects. The effect is in good agreement with the behavior of inulin in dynamic rheological measurements, which approaches the behavior of solid-like materials at higher frequencies, with $\mathrm{G}$ ' being greater than $\mathrm{G}^{\prime \prime}[16]$. At a rice flour hydration level of $70 \%$, the increase ranged from $143 \%$ at $30 \%$ inulin addition to $240 \%$ at a maximum tested inulin level of $66 \%$. In a highly moistened dough ( $110 \%$ by weight of flour), the effect of inulin on $\mathrm{G1}^{\prime}$ decreased due to the negative interaction of inulin and water. There was a low, almost zero effect of oligosaccharides on $G$ 
'at $1 \mathrm{~Hz}$ of gluten-free dough. The observed minor effects may be related to the additional amount of water used in the dough when adding hydrocolloids, with a noticeable effect of water on $G$ 'and with a significant negative interaction of inulin with water. Oligofructose and xanthan gum added separately gave similar linear effects on both modules, resulting in an increase of up to $200 \%$ of the modulus of elasticity when the maximum dosage was added to the dough. The combination of dietary fibers and additional water did not affect the consistency of the dough more than the sum of the individual effects. However, the negative coefficient of interaction between the hydrocoloids used indicates a sharp decrease in the dynamic modules in their presence, especially for the modulus of elasticity, which is probably due to the strong water competition of all hydrocolloids. The frequency scanning showed that for the entire gluten-free dough, the modulus of elasticity, $G^{\prime}$, was greater than the modulus of viscosity, $G^{\prime \prime}$, in the entire frequency range. Both modules increased slightly with frequency. This indicates the firm elastic behavior of all gluten-free dough samples. Therefore, $\tan \delta=\left(\mathrm{G}^{\prime \prime} / \mathrm{G}^{\prime}\right)$ values for all dough samples were below 1 .

The creep recovery tests were also carried out on gluten-free dough samples in the linear viscoelastic region. The voltages ranged from 1.5 to $35 \mathrm{~Pa}$ and were maintained for 60 seconds, which was sufficient for the sample to reach steady state flow. The creep curves of the gluten-free dough show typical viscoelastic behavior, combining viscous liquid and elastic components.

The inclusion of a dietary fiber mixture, an increase in dosage and a decrease in the water content in the dough increased the resistance of the dough to deformation, as evidenced by a decrease in the maximum creep compliance (compliance at the end of the creep phase).

A strong correlation was found between all creep match parameters and the recovery phase equivalents $(p<0.001)$, since creep recovery tests were performed in the linear viscoelastic region. In addition, it was observed that the factors providing a steady state viscosity increase reduced all parameters of creep and compliance during recovery.

The main influence on the creep parameters was related to the water content in the dough. In the absence of hydrocolloids, an increase in water content in the dough resulted in significantly greater instantaneous and delayed elastic compliance (positive linear regression coefficient), which means higher deformation of the dough at constant stress. In contrast, the inclusion of a dietary fiber blend in the formulation reduced instant compliance (negative linear regression coefficient) regardless of the water content of the dough, indicating lower instantaneous elastic deformation in terms of compliance. The dietary fiber mixture dosage increasing when replacing rice flour to $50 \%$ did not show a significant effect on instantaneous deformation in the denser dough (70\% water). However, with more water, replacing $50 \%$ of the rice flour with a fiber blend also reduces elasticity. This fact confirms that different dosages of a mixture of hydrocolloids clearly differ in the requirements for water to strengthen the dough structure.

In empirical studies, the analytical data of Draper-Lin composite samples for dough consistency during direct extrusion and viscous characteristics during mixing were fitted to multiple regression equations using different dosages of dietary fiber mixture as independent variables to evaluate response surfaces of dependent empirical dough quality variables. The stepwise regression equations included only significant coefficients $(p<0.05)$.

The inclusion of fiber blends in different amounts in the rice dough formulation significantly altered the qualitative and quantitative extrusion and viscosimetric characteristics of flour blends with hydrated food fibers. The dependence of extrusion, pasting and gelling parameters on hydrated mixtures of flour and dietary fibers was especially significant for the consistency of both the strength and the peak viscosity area $\left(\mathrm{R}^{2} 0.97\right)$, viscosity at $50{ }^{\circ} \mathrm{C}\left(\mathrm{R}^{2} 0.95\right)$, final viscosity $\left(\mathrm{R}^{2} 0.94\right)$, and complete temperature reduction under cooling $\left(\mathrm{R}^{2} 0.87\right)$. 
Direct extrusion analyses of the prepared rice dough measure the compression force required for the piston disc to squeeze the dough through a predetermined size outlet.

Multivariable processing of rheological variable data provided useful information on significantly correlated viscoelastic characteristics of gluten-free dough samples [17]. Using Pearson's correlation analysis, a correlation coefficient range of 0.43 to 0.99 was obtained for the relationship between the fundamental and empirical properties of rice-based dough matrices with the addition of a mixture of dietary fibers.

These data confirm that either the fundamental rheological parameters recorded at slight deformation or the empirical characteristics covering greater deformation can be useful for characterizing gluten-free dough samples in terms of their viscoelastic behavior in added rice flour and dietary fiber mixtures. The aqueous competition of dietary fiber macromolecules exhibiting different water binding and gelling properties resulted in additive, synergistic and/or antagonistic effects on rheological characteristics. The basic properties of viscoelasticity and the consistency are closely related to the characteristics of the protein network, while the pasteurization/gelling profiles are closely related to starch.

Cookies with $50 \%$ replacement of rice flour with a mixture of dietary fibers showed higher brightness $(\mathrm{L} *)$ among all cookie samples, indicating lighter color, and all cookie samples had red and yellow tinctures (positive values $a *$ and $b *$, respectively) (Table 1 ). In general, the reddish tint was the same for all cookie samples, and the sample containing a mixture of dietary fibers was released with a lower yellow color and higher color saturation $(\mathrm{C} *)$ than the control sample. Cookies with $25 \%$ replacement of rice flour with a mixture of dietary fibers had the same tint as the control sample, but differed from other samples containing the mixture of dietary fibers. Despite the recognized influence of fructans on the color of products, contributing to the Mayar reaction due to the presence of reducing sugars in their chain, the results on color characteristics did not have any definite pattern with an increase in the degree of replacement of rice flour with a mixture of dietary fibers.

Table 1. Physical properties of control biscuits with replacement of rice flour with a mixture of dietary fibres

\begin{tabular}{|l|l|c|c|c|c|}
\hline \multicolumn{2}{|c|}{ Indicators } & Control & $25 \%$ & $50 \%$ & $75 \%$ \\
\hline \multirow{3}{*}{ Color } & $\mathrm{L}^{*}$ & 28.1 & 27.0 & 28.7 & 27,9 \\
\cline { 2 - 6 } & $\mathrm{a}^{*}$ & 9.7 & 9.8 & 9.5 & 9,3 \\
\cline { 2 - 6 } & $\mathrm{b}^{*}$ & 11.8 & 10.8 & 10.5 & 9,3 \\
\cline { 2 - 6 } & $\mathrm{C}^{*}$ & 29.7 & 28.8 & 30.2 & 29,4 \\
\hline Cutting force, $\mathrm{N}$ & 31,8 & 39.5 & 42.8 & 49.2 \\
\hline Bulk volume, $\mathrm{sm}^{3} / \mathrm{g}$ & 2,2 & 2.3 & 1.8 & 1.5 \\
\hline Liquescence coefficient & 0,7 & 0.8 & 0.8 & 0.7 \\
\hline
\end{tabular}

As for the cutting strength, the biscuit samples with the dietary fiber mixture had higher values than the control biscuit sample, but they were similar to each other (Table 1). High sugar cookies may have higher hardness due to crystallization of the sugar during cooling. Although the amount of sugar was the same for all samples, it is likely that the addition of the dietary fiber mixture resulted in increased cutting strength due to fructose crystallization.

Cookies with $25 \%$ replacement of rice flour with a mixture of dietary fibers had a specific volume substantially the same as the control sample, while samples with 50 and $75 \%$ replacement of rice flour with a mixture of dietary fibers had a lower specific volume than 
the control sample (Table 1). With respect to the blur ratio, the $25 \%$ and $50 \%$ replacement cookie samples had an increase in the diameter of the dough preform greater than the control sample. A higher amount of fructose in samples of cookies containing a mixture of dietary fibers may explain the fact that these samples of cookies had a higher melt ratio than the control sample of cookies except for a sample with $75 \%$ replacement of rice flour with a mixture of dietary fibers, which is likely due to increased caramelization during baking.

\section{References}

1. A. Kaur, O. Shimoni, M. Wallach,. J. Gastroenterol., 52, 1001-1012 (2017) https://doi.org/10.1007/s00535-017-1357-7

2. I.Rybicka, K. Doba, O. Bińczak, Int. J. Food Sci. Technol., 54(9), 2661-2667 (2019) https://doi.org/10.1111/ijfs.14190

3. M.Elleuch, D.Bedigian, O.Roiseux, S. Besbes, C. Blecker, H. Attia, Food Chem., 124(2), 411-421 (2011) https://doi.org/10.1016/j.foodchem.2010.06.077

4. J. Xu, Y. Li, Y. Zhao, D. Wang, W. Wang, J. Func. Foods, 80, 104434 (2021) https://doi.org/10.1016/j.jff.2021.104434

5. V.D. Capriles, J.A.G. Arêas, Food Func., 4, 104-110 https://doi.org/10.1039/c2fo10283h

6. D. Mohanty, S. Misra, S.Mohapatra, P.S. Sahu, Food Biosci., 26, 152-160 (2018) https://doi.org/10.1016/J.FBIO.2018.10.008

7. D. Farias, F. Araújo, I. Neri Numa, G. Pastore, Trends Food Sci. Techn., 93, 23-35 (2019) https://doi.org/10.1016/j.tifs.2019.09.004

8. M. Shoaib, A. Shehzad, M. Omar, A. Rakha, H. Raza, H.R. Sharif, A. Shakeel, A. Ansari, S. Niazi, Carbohyd. Polym., 147, 444-454 (2016) https://doi.org/10.1016/i.carbpol.2016.04.020

9. T. F. da Silva, A.C. Conti-Silva, LWT - Food Sci. Techn., 90, 172-179 (2018) https://doi.org/10.1016/j.lwt.2017.12.031

10. A.S. Althubiani, S.B. Al-Ghamdi, Samreen, F.A. Qais, M.S. Khan, H.A.Malak, In: M.S.A. Khan, I. Ahmad, D. Chattopadhyay (eds) New Look to Phytomedicine, 63-88 (Academic Press, 2019) https://doi.org/10.1016/B978-0-12-814619-4.00004-5

11.C. Morris, G.A. Morris, Food Chem., 133, 237-248 http://doi.org/10.1016/j.foodchem.2012.01.027

12. A.K. Strelkova, I.B. Krasina, K.N. Storcheus, E.V. Filippova, News of Institutes of $\begin{array}{lllll}\text { Higher Education. Food Technology, 1(379), 82-84 (2021) } & \end{array}$ https://doi.org/10.26297/0579-3009.2021.1.19

13. A. Zoghi, R.S. Mirmahdi, M. Mohammadi, Inter. J. Food Sci. Techn., 56, 3138-3147 (2020) http://doi.org/10.1111/ijfs.14887

14. S. Benkadri, A. Salvador, T. Sanz, M. Nasreddine Zidoune Foods, 10, 12 (2021) https://doi.org/10.3390/foods10010012

15. A.K. Strelkova, I.B. Krasina, E.V. Filippova, A.V. Lysenko, News of Institutes of Higher Education. Food Technology, 2-3(380-381), 45-49 (2021) https://doi.org/10.26297/05793009.2021.2-3.11

16. F. Ronda, S. Pérez-Quirce, A. Angioloni, C. Collar, Food Hydrocoll., 32(2), 252-262 (2013) http://doi.org/10.1016/j.foodhyd.2013.01.014

17. S. Benkadri, A. Salvador, M.N. Zidoune, T. Sanz, Food Sci. Technol. Int., 24, 607-616 (2018) http://doi.org/10.1177/1082013218779323 